\title{
Elevated corticosterone in feathers correlates with corticosterone-induced decreased feather quality: a validation study
}

\author{
Christine R. Lattin, J. Michael Reed, David W. DesRochers and L. Michael Romero \\ C. R. Lattin (christine.lattin@tufts.edu), J. M. Reed and L. M. Romero, Dept of Biol., Tufts Univ., 163 Packard Ave., Medford, MA 02155, \\ USA. - D. W. DesRochers, Dept of Natural Sci., Dalton State Coll. 650 Coll. Drv., Dalton, GA 30720, USA.
}

\begin{abstract}
The newly described technique of extracting corticosterone (CORT) from bird feathers may serve as a less invasive, more integrated measure of a bird's stress response. Previous work indicated that elevated plasma CORT resulted in poorer quality feathers during molt. We tested the hypothesis that a direct link exists between plasma and feather CORT concentrations. We experimentally increased plasma CORT concentrations using implants and found that the corresponding rise in CORT could be detected in feathers grown during implantation. Furthermore, CORT levels in two feathers grown at the same time from the same bird were very consistent. These results provide evidence that elevated CORT is a causative factor in decreasing feather quality during molt. However, there remain technical details that suggest caution when interpreting data from CORT extracted from feathers. Different portions of a growing feather did not necessarily reflect changes in plasma CORT at the time different parts of the feather were forming, a standard pool of homogenized feathers indicated that sample mass affects measured feather CORT concentration, and different antibodies produced different measured CORT concentrations, leaving in doubt the exact steroid being assayed.
\end{abstract}

In birds, the stress response consists of a suite of behavioral and physiological changes that help animals cope with stressors such as bad weather, predators, food shortages and social instability (Wingfield and Romero 2001, Wingfield et al. 1997). One important response to these stressors is activation of the hypothalamus-pituitary-adrenal axis, resulting in increased synthesis and release of corticosterone (CORT; Sapolsky et al. 2000). Both baseline and stressinduced plasma CORT vary seasonally in a predictable pattern in many species of birds with CORT typically being low during the prebasic molt (Romero 2002). Because one of CORT's physiological roles is breaking down protein (Sapolsky et al. 2000), and feathers are primarily made up of the protein keratin, high plasma CORT concentrations could interfere with the production of high-quality feathers essential for flight and insulation. Indeed, DesRochers et al. (2009) showed that birds with higher levels of circulating endogenous and exogenous CORT had lighter feathers with altered micro-structure, which in some cases led to reduced feather strength, compared with control birds.

The most widely used technique for measuring CORT is by sampling of blood, an invasive "snapshot" of an animal's CORT concentration that is difficult to extrapolate to a long-term profile. Consequently, other techniques for measuring CORT have been developed for birds, most notably measuring fecal glucocorticoid metabolites (FGMs, e.g., Mostl et al. 2005), and, more recently, CORT in bird feathers (Bortolotti et al. 2008, 2009). There are a number of studies using mass spectrometry to show that steroid hormones and their metabolites can be directly incorporated into the protein matrix of mammalian hair (for example, see Cirimele et al. 2000), so it makes sense that these hormones might also be found in bird feathers. And indeed, extracting CORT from bird feathers holds promise for addressing new kinds of questions, as early work has shown (Bortolotti et al. 2008, 2009).

The primary purpose of this study was to test the hypothesis that birds with experimentally increased CORT levels grow feathers that contain significantly more CORT than feathers from control birds. From CORT-implanted birds, re-grown feathers grow slower (Romero et al. 2005) and are of lower quality (DesRochers et al. 2009) than feathers re-grown from sham-implanted birds. Bortolotti et al. (2009) showed that feathers with visible deformities known as fault bars contained significantly more CORT than did adjacent feather sections lacking these bars. However, the use of CORT implants by DesRochers et al. (2009) did not result in fault bars and the differences in feather quality between sham- and CORT-implanted birds were more subtle. Our first goal was to determine if a five-day exogenous increase in plasma CORT was detectable in feathers.

Our second goal in this experiment was to continue validation work for the technique of measuring CORT in feathers. All techniques for measuring CORT to date have limitations (e.g. Millspaugh and Washburn 2004), so continued validation work is necessary for establishing the benefits and limitations of measuring CORT in feathers. 
Consequently, we tested four inferences inherent in extracting CORT from bird feathers. First, although variation in the amount of CORT in different feathers may reflect variation in plasma CORT levels while the feathers were growing (Bortolotti et al. 2008, 2009), it may also reflect measurement error. We used a standard pool of homogenized feathers, rather than a plasma standard (Bortolotti et al. 2008), to provide a more appropriate assessment for intra- and inter-assay variation in feather samples and thus to better distinguish measurement error from true variation. Second, we used re-grown feathers with defined periods of CORT exposure to test whether CORT levels in feather sections reflected plasma CORT at the time different parts of the feather were forming, as suggested by Bortolotti et al. (2009). Third, we tested whether CORT concentrations were consistent among feathers growing at the same time. High consistency among feathers is essential if one or a small number of feathers is assumed to represent circulating CORT concentrations in an individual during molt. Finally, we tested whether different antibodies measured similar levels of CORT in the feathers.

\section{Material and methods}

\section{Feather sources}

Feathers were obtained from wild-caught European starlings Sturnus vulgaris from Newbury, Massachusetts, USA, and housed in the laboratory for several months. Birds were fed ad libitum on "Purina Start and Grow", a high-protein commercial bird food. Feathers from red-tailed hawks Buteo jamaicensis came from wild birds treated by the Tufts Wildlife Veterinary Clinic (North Grafton, Massachusetts, USA). In all cases, we only used flight feathers. All experiments were performed in accordance with AALAC procedures and approved by the Tufts University Institutional Animal Care and Use Committee.

\section{Assay protocol}

Feathers were prepared and assayed following Bortolotti et al. (2008), with some modifications. Briefly, we removed the calamus and minced the rachis and feather vanes into pieces $<5 \mathrm{~mm}^{2}$. We then used methanol to extract CORT from feathers. Steroid hormones are generally highly soluble in lower alcohols such as methanol (Pötsch and Moeller 1996). Minced feather was mixed with $7 \mathrm{ml}$ of methanol (HPLC grade, Fisher Scientific), placed in a sonicating water bath at room temperature for $30 \mathrm{~min}$, then incubated overnight in a shaking $50^{\circ} \mathrm{C}$ water bath. Methanol was separated from feather using vacuum filtration, using \#4 Whatman filter paper in a filtration funnel. Feather bits, the sample vial, and the filter paper were washed twice with approximately $2.5 \mathrm{ml}$ of additional methanol with washes added to the methanol extract. Methanol extracts were dried under nitrogen gas in a $50^{\circ} \mathrm{C}$ water bath. Extracts were reconstituted in PBS buffer and run through a standard radioimmunoassay (RIA) as originally described by Wingfield et al. (1992). The efficiency of methanol extraction was determined by initially spiking each sample with a small amount of 3H-CORT. Approximately
$70-80 \%$ of this radioactivity was recovered in reconstituted samples; this is lower than the $>90 \%$ recoveries reported by Bortolotti et al. (2008), probably due to differences in filtration technique. All final values were adjusted by recovery percentages. Samples were normalized by feather length; Bortolotti et al. (2008, 2009) suggested this was preferable to standardizing by mass because of the way feathers grow and elongate along their radial circumference. Standard samples of powdered homogenized feather (see next section) were normalized by mass.

Two different anti-CORT antibodies were used in the RIA (Table 1). The first was from Endocrine Sciences/ Esoterix (B3-163, Calabasas Hills, California, USA, lot $404 \mathrm{~A}$ ), and has been used by our lab for a number of years to measure CORT in several species, including starlings (e.g., Cyr et al. 2008). The second was obtained from Sigma-Aldrich (C 8784, Saint Louis, Missouri, USA, lot $57 \mathrm{~K} 4791)$ and is the same antibody used by Bortolotti et al. (2008).

\section{Creating a feather CORT standard}

We created a homogenous mixture of pooled feathers to assess inter-assay variation and examine methanol extraction efficiency with different amounts of feather. We prepared 138 European starling feathers from four different individuals by removing the calamus, cutting feathers into small bits, and pulverizing them in a Ball mill (Kleco model 4200, Visalia, California, USA) until reduced to dust. This dust was then mixed thoroughly.

We ran four iterations of the feather assay using different amounts of pulverized feather (measured by mass), ranging from $3 \mathrm{mg}$ (equivalent to approximately an eighth of a single starling primary feather) to $99 \mathrm{mg}$ (equivalent to approximately four complete primary feathers). We ran all assays on pulverized feather as described above. Sample mass was found to affect CORT concentration (see results), so we only used samples of the same mass to determine inter-assay variation. Because each individual sample was run in duplicate, intra-assay variation was calculated as the

Table 1. Steroid cross-reactivity of two different antisera used in radioimmunoassays with feather extracts. Cross-reactivity information was supplied by manufacturers on request; n.r. =not reported. Cross-reactivity with all other hormones tested was $<1 \%$.

\begin{tabular}{lcc}
\hline Cross-reactant & Percent cross-reactivity \\
\cline { 2 - 3 } & $\begin{array}{c}\text { Endocrine } \\
\text { sciences/esoterix } \\
\text { antibody }\end{array}$ & $\begin{array}{c}\text { Sigma } \\
\text { antibody }\end{array}$ \\
\hline Aldosterone & 0.2 & \\
Androstenedione & $\mathrm{n} . \mathrm{r}$. & 4.4 \\
Cortisol & 0.4 & 2.6 \\
Cortisone & $<0.1$ & 4.5 \\
Dehydroepiandrosterone & 0.03 & 3.2 \\
11-deoxycorticosterone & 3.3 & $<10$ \\
$5 \alpha$-dihydrotestosterone & $\mathrm{n} . \mathrm{r}$. & 20 \\
Estradiol & $<0.01$ & 1.4 \\
17-hydroxyprogesterone & 0.2 & $<30$ \\
20 $\alpha$-hydroxyprogesterone & 0.2 & 1.8 \\
20ß-hydroxyprogesterone & 0.2 & 8.8 \\
Progesterone & 0.6 & 5.2 \\
Testosterone & 0.1 & 15.7 \\
\hline
\end{tabular}


mean variation in CORT concentration between duplicates. In these four runs of the assay, intra-assay variation was $6.9 \%$. Inter-assay variation was calculated as the mean variation in CORT concentration for samples of the same mass among all four runs of the assay, and was $40.4 \%$. To analyze the relationship between sample mass and measured CORT concentration in these assays, we used Tablecurve 2D (Systat Software, Inc.) and compared models using Akaike's Information Criterion calculated using general linear models in SAS (SAS Institute, version 9.1.3).

\section{CORT and sham implant experiments}

We plucked 26 flight feathers each from 20 captive European starlings to simulate molt and elicit feather growth (for more details, see Strochlic and Romero 2008, and DesRochers et al. 2009). Fourteen days later, when the synchronously re-growing feathers were approximately $1 / 3$ re-grown, we subcutaneously implanted silastic capsules between the shoulder blades of each bird. Nine birds received capsules containing crystalline CORT (Sigma Chemical Co.), while the other 11 received empty capsules. Five days after implantation, all capsules were removed. Feathers were marked with permanent marker when implants were inserted and again when implants were removed, in order to differentiate among parts of the feather that grew before, during and after implantation. Mean feather length was $61 \mathrm{~mm}$; mean feather growth during the five days of implantation was $25 \mathrm{~mm}$. We collected blood samples from each bird before implantation, and three and five days after implantation. All surgical and blood sampling procedures followed Romero et al. (2005); plasma RIA was conducted following Wingfield et al. (1992). The birds from this experiment were also part of a different study on feather quality that has already been reported (DesRochers et al. 2009) and the plasma CORT values resulting from the implants are reported there. However, there was no overlap between the feathers used in this and the previous study.

Feathers from these birds were processed in two different ways. In one assay, right secondary 1 from each bird was divided into three parts: before, during, and after implantation, with each part run as a separate sample in the same assay. Because of the way feathers grow-at a given point along the rachis, all parts from the edge of the inner vane to the edge of the outer vane grow at the same time (Bortolotti et al. 2009), we cut feather sections perpendicular to the rachis. In a second assay, left secondary 1 and 2 from each bird were run as whole-feather samples. In all cases, feathers or feather sections were measured, weighed and minced as described above, then run through the feather RIA. In these assays, inter-assay variation was measured using a plasma standard. Inter- and intra-assay variation was $9 \%$ and $6.5 \%$, respectively. We analyzed data obtained from these assays using repeated-measures ANOVA in SPSS (Student ver. 16.0 for Windows, SPSS Inc., 2008), using feather section (before, during, or after implantation) or feather type (left secondary 1 or 2 ) as the repeated measure. The Greenhouse-Geisser correction for heteroscedasticity was applied when appropriate. In assays using pulverized pooled feather, we saw a large effect of sample mass on CORT concentration (see Results), but in these two assays there was very little difference in mass across samples, and so mass did not have a significant relationship with CORT concentrations and was not included as a covariate in our statistical models.

\section{Results}

The two antibodies used in this study produced different results. While we obtained values in the range of $2.4 \mathrm{pg}$ CORT $/ \mathrm{mm}$ feather (for red-tailed hawk) to $6.7 \mathrm{pg}$ CORT/ $\mathrm{mm}$ feather (for European starling) using the Sigma antibody, we were unable to detect CORT in feathers using the Endocrine Sciences antibody, even when samples consisted of five European starling feathers minced together. Hence, for the rest of the experiments reported here, we only used the Sigma antibody.

With pulverized pooled European starling feather as a CORT standard, CORT/mg of feather decreased significantly with increasing amount of feather used (Fig. 1). The best-fit model for the data from the four runs of the assay was significantly curvilinear, described by the line $y=$ $-8.20+(80.41 / \ln \mathrm{x})$, where $\mathrm{x}$ is feather mass $(\mathrm{mg})$ and $\mathrm{y}$ is CORT/mg $\left(r^{2}=0.83\right)$. This relationship was a statistically significantly better fit than was a horizontal line (the null model; $\triangle \mathrm{AIC}=-49.8$ ).

When comparing feathers from CORT and sham implanted birds, more CORT was detected from birds receiving CORT implants than in those receiving sham implants (Fig. 2; $\mathrm{F}_{1,18}=33.6, \mathrm{P}<0.0001$ ). When feathers were divided into three sections (before, during, and after implantation), the amount of CORT differed across feather sections $\left(\mathrm{F}_{2,28}=9.1, \mathrm{p}=0.002\right)$, although patterns did not differ between CORT- and sham-implanted birds $\left(\mathrm{F}_{2,28}=\right.$ $0.4, \mathrm{p}=0.6$ ). Furthermore, CORT levels in the different feather sections did not correspond to plasma CORT during the same time periods, where CORT-implanted birds showed a significant increase in plasma CORT only while implants were in place (see DesRochers et al. 2009 for graph and statistical analysis).

When left secondary 1 and 2 were compared from each bird, more CORT was detected again in feathers from

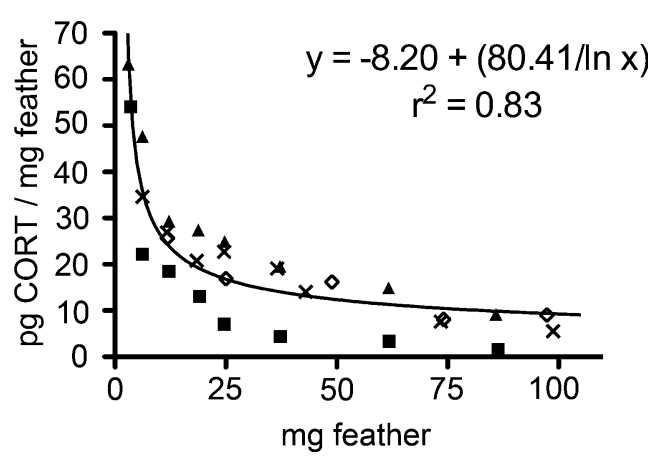

Figure 1. The relationship between sample mass (mg) and concentration of corticosterone (pg CORT/mg feather) in samples of pooled pulverized feathers from European starlings. Four different runs of the assay are represented on the graph, with different symbols used for each. The best-fit line equation for the data is shown. 


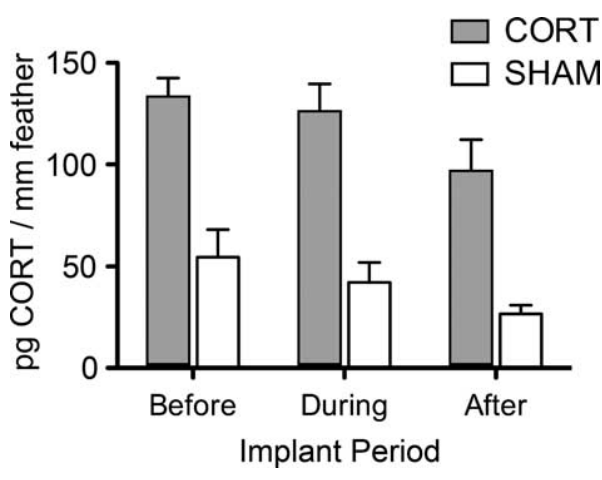

Figure 2. Amount of corticosterone (CORT) detected in minced feathers from European starlings receiving CORT $(n=9)$, or empty (sham, $\mathrm{n}=11$ ) silastic implants. Feathers were induced to grow through plucking, and $14 \mathrm{~d}$ later, implants were inserted subcutaneously. Five days later, implants were removed. Feathers were marked when implants were put in and again when implants were removed, allowing differentiation among parts of the feather that grew before, during and after implantation.

CORT-implanted birds compared to those receiving sham implants (Fig. 3; $\mathrm{F}_{1,18}=12.2, \mathrm{p}=0.003$ ). There was no difference in CORT levels between feathers from the same bird $\left(\mathrm{F}_{1,18}=0.1, \mathrm{p}=0.75\right)$.

\section{Discussion}

Results clearly indicate that higher CORT levels were detected in the feathers of birds whose plasma CORT levels were experimentally increased. Other feathers from these CORT-implanted birds were shown to have lower mass, altered micro-structure, and weaker barbicel hooking strength compared with feathers from sham-implanted birds (DesRochers et al. 2009). This shows that increased plasma CORT during molt can result in both lower feather quality and an increase in feather CORT, even when birds have ad libitum access to high-protein food. This suggests that the increased CORT is the causative agent in creating lower feather quality. However, CORT might not be the only causative agent. An induced molt could have led to faster feather growth, which can cause the growth of

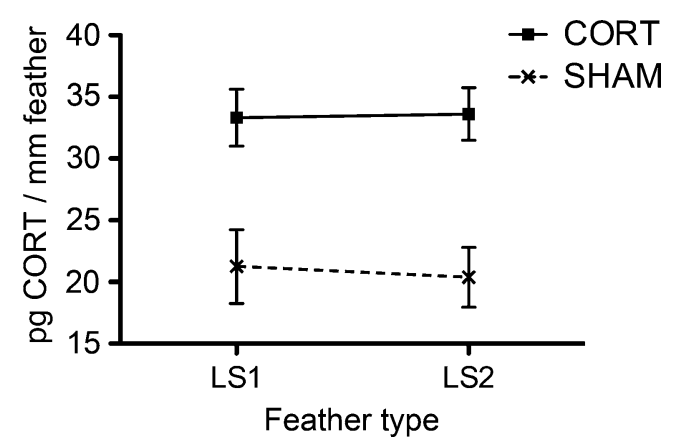

Figure 3. Amount of corticosterone (CORT) detected in minced feathers from European starlings receiving CORT $(n=9)$ or empty (sham, $\mathrm{n}=11$ ) silastic implants. Feathers were induced to grow through plucking, and 14 days later, implants were inserted subcutaneously. Five days later, implants were removed. The two feathers compared in each bird were left secondaries 1 (LS1) and 2 (LS2). feathers that can have lower mass (Dawson 2004), and shorter length (Hall and Fransson 2000) than normal. Therefore, it remains to be determined whether increased feather CORT is generally indicative of lower-quality feathers, as measured by structural characteristics and mechanical properties.

Earlier work interpreted variation in the amount of CORT per unit length for different sections of the feather as deriving from day-to-day variation in plasma CORT resulting from responses to stressors during feather growth (Bortolotti et al. 2009). The fact that CORT-filled implants resulted in higher CORT throughout the feather, even though plasma CORT was only elevated during implantation, suggests caution in using this interpretation. Feather CORT did not reflect plasma CORT on a fine sectionby-section time scale in our study, but it is difficult to reconcile this result with what is known about the mechanics of feather growth. It is unknown how and when CORT is deposited in feathers. CORT may be incorporated into the growing feather from blood supplying it during feather growth and keratinization, it may be externally deposited (for example via preen oils, or diffusion from the skin surrounding the feather), or both, as seems to be the case with hair (Thieme et al. 2003). If CORT were incorporated into the growing feather only from blood, we would expect to see the highest feather CORT in the portion of the feather that stays vascularized the longest the proximal portion. However, the pattern of CORT deposition in the feathers of both sham- and CORTimplanted birds is the opposite: highest CORT in the most distal portions of the feather, lowest CORT in the most proximal sections.

It is certainly possible that the sustained elevation in CORT resulting from the implants produced an abnormal deposition of CORT in the feathers. It should be remembered that birds typically regulate CORT secretion during molt (Romero 2002), presumably to prevent high titers of CORT such as those induced by the implants (Romero et al. 2005). The high CORT titers from the implants was previously interpreted to have exposed the underlying physiological trade-off leading to lower feather quality (Strochlic and Romero 2008) and the implants may have similarly short-circuited normal CORT deposition in the feathers. However, from this one would predict lower CORT levels in feathers before CORT implants, and the reverse was observed.

The implant-induced CORT variation in different portions of a growing feather provides some support to the idea that CORT is externally deposited, perhaps via preen oil, as the distal portion of feathers is the oldest and is subject to the most preening. However, there is no evidence for the presence of CORT in avian preen oils. For example, washing feathers in a dilute soap solution did not alter feather CORT, although it should be noted that a hexane wash did result in lower feather CORT (Bortolotti et al. 2008). Also, our lab failed to extract CORT from European starling preen oil using methanol extraction and both the Endocrine Sciences and Sigma antibodies (unpublished data). In any case, it makes clear that until we know more about how CORT is deposited in feathers, we should use caution in assuming that CORT from feather sections is a 
stand-in for relative CORT concentrations in the plasma while those sections were growing.

We expected that any antibody that binds well to CORT in a plasma RIA could also be used in a feather RIA, assuming that unmodified CORT is deposited in feathers. We were therefore surprised that the Endocrine Sciences antibody detected no CORT from feather extracts. The different results between the Sigma and Endocrine Sciences antibodies could reflect a lower sensitivity of the Endocrine Sciences antibody; CORT levels in feathers are an order of magnitude lower than those detected in plasma, and the manufacturer-reported affinity constant of the Sigma antibody is seven times greater than that of the Endocrine Sciences antibody $\left(35 \times 10^{9} \mathrm{l} / \mathrm{mol}\right.$ and $7 \times 10^{9} \mathrm{l} / \mathrm{mol}$, respectively). Alternatively, the Sigma antibody might be binding to something other than unmodified CORT. Although sulfonated and glucuronidated CORT metabolites in feather extracts are not detected by the Sigma antibody (Bortolotti et al. 2008), the antibody could crossreact with one of the other metabolites of CORT, or potentially with other steroid hormones as recently shown in bird egg yolks (Rettenbacher et al. 2009). Indeed, information provided by Sigma indicates that their antibody has much higher cross-reactivity than the Endocrine Sciences antibody, especially with steroids like progesterone, 11-deoxycorticosterone and testosterone. Although work to date suggests that CORT is indeed being measured in feather extracts, our results from the Endocrine Sciences antibody suggests caution. Although it was beyond the scope of this project, using high-performance liquid chromatography and/or gas chromatography would better quantify the components of feather extracts, and injecting radiolabeled CORT during feather growth would further elucidate which hormones or hormone metabolites are deposited in feathers.

Our pooled homogenized feather standard should have resulted in a constant CORT per unit weight. However, the decrease with increasing sample mass is similar to results reported in fecal glucocorticoid metabolite (FGM) studies (e.g., Millspaugh and Washburn 2004, Tempel and Gutierrez 2004), which also use methanol-based extraction. A greater concentration of methanol per unit sample mass could lead to better extraction of CORT and/or its metabolites (Millspaugh and Washburn 2004). For example, in a recent paper using ethanol extraction of thyroid hormones from feces, Wasser et al. (2010) showed ethanol extraction was less effective as the volume/mass ratio fell below $15 \mathrm{ml}$ ethanol $/ 0.1 \mathrm{~g}$ feces.

Because both feather and fecal samples below $20 \mathrm{mg}$ show the most dramatic increase in CORT/FGM concentrations, avoiding small sample mass could also solve this problem, but would prevent analyses of small portions of feathers or feathers from small species. Another approach could involve standardizing the mass of all samples compared in an assay. Because the relationship between sample mass and CORT concentration seems consistent (even across FGM and feather CORT studies), this suggests that a species-specific curve of sample mass vs. CORT concentration could be used to standardize sample mass or that sample mass could be included as a covariate in analyses. Also, a recent paper addressing this issue in FGM assays found that just using a more accurate scale to weigh very small samples removed a lot of variation (Hayward et al. 2010). What is clear, however, is that samples of different mass (especially below $20 \mathrm{mg}$ ) are not directly comparable.

In conclusion, the present data provide interesting evidence that increased plasma CORT can result both in decreased feather quality and in increased feather CORT. However, some important technical constraints must be considered when using this feather assay, including variation in the effectiveness of different antibodies and the effect of sample mass on measured feather CORT concentration. Investigators should also be wary of trying to use different portions of a growing feather to reflect changes in plasma CORT. Further studies should focus on how CORT is deposited in feathers, as well as exploring various additional potential sources of variation such as the effects of feather melaninization (Almasi et al. 2008, Roulin et al. 2008).

Acknowledgements - We thank J. Awerman for help implanting birds with silastic capsules and M. Pokras for providing hawk feathers. Funding was provided by grant IOB-0542099 from the U.S. National Science Foundation (to LMR). We thank J. Williams and two anonymous reviewers for useful comments on the manuscript.

\section{References}

Almasi, B., Roulin, A., Jenni-Eiermann, S. and Jenni, L. 2008. Parental investment and its sensitivity to corticosterone is linked to melanin-based coloration in barn owls. - Horm. Behav. 54: 217-223.

Bortolotti, G. R., Marchant, T. A., Blas, J. and German, T. 2008. Corticosterone in feathers is a long-term integrated measure of avian stress physiology. - Funct. Ecol. 22: 494-500.

Bortolotti, G. R., Marchant, T. A., Blas, J. and Cabezas, S. 2009. Tracking stress: localisation, deposition and stability of corticosterone in feathers. - J. Exp. Biol. 212: 1477-1482.

Cirimele, V., Kintz, P., Dumestre, V., Goullé, J. P. and Ludes, B. 2000. Identification of ten corticosteroids in human hair by liquid chromatography-ionspray mass spectrometry. - Forensic Sci. Internat. 107: 381-388.

Cyr, N. E., Wikelski, M. and Romero, L. M. 2008. Increased energy expenditure but decreased stress responsiveness during molt. - Physiol. Biochem. Zool. 81: 452-462.

Dawson, A. 2004. The effects of delaying the start of moult on the duration of moult, primary feather growth rates and feather mass in common starlings Sturnus vulgaris. - Ibis 146: 493500.

DesRochers, D. W., Reed, J. M., Awerman, J., Kluge, J. A., Wilkinson, J., van Griethuijsen, L. I., Aman, J. and Romero, L. M. 2009. Exogenous and endogenous corticosterone alter feather quality. - Comp. Biochem. Physiol. A: Mol. Integr. Physiol. 152: 46-52.

Hall, K. S. S. and Fransson, T. 2000. Lesser whitethroats under time-constraint moult more rapidly and grow shorter wing feathers. - J. Avian Biol. 31: 583-587.

Hayward, L. S., Booth, R. K. and Wasser, S. K. 2010. Eliminating the artificial effect of sample mass on avian fecal hormone metabolite concentration. - Gen. Comp. Endocrinol. 169: 117-122.

Millspaugh, J. J. and Washburn, B. E. 2004. Use of fecal glucocorticoid metabolite measures in conservation biology 
research: considerations for application and interpretation.. - Gen. Comp. Endocrinol. 138: 189-199.

Mostl, E., Rettenbacher, S. and Palme, R. 2005. Measurement of corticosterone in birds' droppings: an analytical approach. - Ann. N. Y. Acad. Sci. 1046: 17-34.

Pötsch, L. and Moeller, M. R. 1996. On pathways for small molecules into and out of human hair fibers. - J. Forensic Sci. 41: 121-125.

Rettenbacher, S., Mostl, E. and Groothuis, T. G. G. 2009. Gestagens and glucocorticoids in chicken eggs. - Gen. Comp. Endocrinol. 164: 125-129.

Romero, L. M. 2002. Seasonal changes in plasma glucocorticoid concentrations in free-living vertebrates. - Gen. Comp. Endocrinol. 128: 1-24.

Romero, L. M., Strochlic, D. E. and Wingfield, J. C. 2005. Corticosterone inhibits feather growth: potential mechanism explaining seasonal down regulation of corticosterone during molt. - Comp. Biochem. Physiol. A: Mol. Integr. Physiol. 142: 65-73.

Roulin, A., Almasi, B., Rossi-Pedruzzi, A., Ducrest, A. L., Wakamatsu, K., Miksik, I., Blount, J. D., Jenni-Eiermann, S. and Jenni, L., 2008. Corticosterone mediates the conditiondependent component of melanin-based coloration. - Anim. Behav. 75: 1351-1358.

Sapolsky, R. M., Romero, L. M. and Munck, A. U. 2000. How do glucocorticoids influence stress responses? Integrating permissive, suppressive, stimulatory, and preparative actions. - Endocr. Rev. 21: 55-89.

Strochlic, D. E. and Romero, L. M. 2008. The effects of chronic psychological and physical stress on feather replacement in
European starlings (Sturnus vulgaris). - Comp. Biochem. Physiol. A: Mol. Integr. Physiol. 149: 68-79.

Tempel, D. J. and Gutierrez, R. J. 2004. Factors related to fecal corticosterone levels in California spotted owls: Implications for assessing chronic stress. - Conserv. Biol. 18: 538-547.

Thieme, D., Anielski, P., Grosse, J., Sachs, H. and Mueller, R. K. 2003. Identification of anabolic steroids in serum, urine, sweat and hair: comparison of metabolic patterns. - Analytica Chimica Acta 483: 299-306.

Wasser, S. K., Azkarate, J. C., Booth, R. K., Hayward, L. S., Hunt, K., Ayres, K., Vynne, C., Gobush, K., Canales-Espinosa, D. and Rodríguez-Luna, E. 2010. Non-invasive measurement of thyroid hormone in feces in a diverse array of avian and mammalian species. - Gen. Comp. Endocrinol. 168: 1-7.

Wingfield, J. C. and Romero, L. M. 2001. Adrenocortical responses to stress and their modulation in free-living vertebrates. - In: McEwen, B.S., Goodman, H.M (eds), Handbook of physiology; section 7: the endocrine system; volume IV: coping with the environment: neural and endocrine mechanisms. Oxford Univ. Press, pp. 211-234.

Wingfield, J. C., Vleck, C. M. and Moore, M. C. 1992. Seasonal changes in the adrenocortical response to stress in birds of the Sonoran Desert. - J. Exp. Zool. 264: 419-428.

Wingfield, J. C., Hunt, K., Breuner, C., Dunlap, K., Fowler, G. S., Freed, L. and Lepson, J. 1997. Environmental stress, field endocrinology, and conservation biology. - In: Clemmons, J. R. and Buchholz, R. (eds), Behavioral approaches to conservation in the wild. Cambridge Univ. Press, pp. 95-131. 